\title{
A Comparative Study of Some Pseudorandom Number Generators
}

\author{
I. Vattulainen ${ }^{1}$, K. Kankaala ${ }^{1,2}$, J. Saarinen ${ }^{1}$, and T. Ala-Nissila ${ }^{1,3}$ \\ ${ }^{1}$ Department of Electrical Engineering \\ Tampere University of Technology \\ P.O. Box 692 \\ FIN - 33101 Tampere, Finland \\ ${ }^{2}$ Centre for Scientific Computing \\ P.O. Box 405, FIN - 02100 Espoo, Finland \\ ${ }^{3}$ Research Institute for Theoretical Physics \\ P.O. Box 9 (Siltavuorenpenger 20 C) \\ FIN - 00014 University of Helsinki, Finland
}

\begin{abstract}
We present results of an extensive test program of a group of pseudorandom number generators which are commonly used in the applications of physics, in particular in Monte Carlo simulations. The generators include public domain programs, manufacturer installed routines and a random number sequence produced from physical noise. We start by traditional statistical tests, followed by detailed bit level and visual tests. The computational speed of various algorithms is also scrutinized. Our results allow direct comparisons between the properties of different generators, as well as an assessment of the efficiency of the various test methods. This information provides the best available criterion to choose the best possible generator for a given problem. However, in light of recent problems reported with some of these generators, we also discuss the importance of developing more refined physical tests to find possible correlations not revealed by the present test methods.
\end{abstract}

PACS numbers: 02.50.-r, 02.50.Ng, 75.40.Mg.

Key words: Randomness, random number generators, Monte Carlo simulations. 


\section{Introduction}

"Have you generated any new random numbers today?"

J. КӒРYAHO

Long sequences of random numbers are currently required in numerous applications, in particular within statistical mechanics, particle physics, and applied mathematics. The methods utilizing random numbers include Monte Carlo simulation techniques [5], stochastic optimization [1], and cryptography [6, 12, 61], all of which usually require fast and reliable random number sources. In practice, the random numbers needed for these methods are produced by deterministic rules, implemented as (pseudo)random number generator algorithms which usually rely on simple aritmethic operations. By their definition, the maximum - length sequences produced by all these algorithms are finite and reproducible, and can thus be "random" only in some limited sense [8, 9].

Despite the importance of creating good pseudorandom number generators, fairly little theoretical work exists on random number generation. Thus, the properties of many generators are not well understood in depth. Some random number generator algorithms have been studied in the general context of cellular automata [8], and deterministic chaos [4]. In particular, number theory has yielded exact results on the periodicity and lattice structure for linear congruential and Tausworthe generators [11, 40, 30, 55]. These results have led to theoretical methods of evaluating the algorithms, the most notable being the so called spectral test. However, most of these theoretical results are derived for the full period of the generator while in practice the behavior of subsequences of substantially shorter lengths is of particular importance in applications. In addition, the actual implementation of the random number generator algorithm may affect the quality of its output. Thus, in situ tests of implemented programs are usually needed.

Despite this obvious need for in situ testing of pseudorandom number generators, only relatively few authors have presented results to this end [32, 33, 49, 42]. Most likely, there are two main reasons for this. The first is the persistence of underlying fundamental problems in the actual definitions of "randomness" and "random" sequences which have given no unique practical recipe for testing a finite sequence of numbers [31]. Thus various authors have developed an array of different tests which mostly probe some of the statistical properties of the sequences, or test correlations e.g. on the binary level. Recently, Compagner and Hoogland [8] have presented a 
somewhat more systematic approach to randomness as embodied in finite sequences. They propose testing the values of all possible correlation coefficients of an ensemble of a given sequence and all of its "translations" (iterated variations) [10], a task which nevertheless appears rather formidable for practical purposes. We are not aware of any attempts to actually carry out their program. The second reason is probably more practical, namely the gradual evolution of improved pseudorandom number generator algorithms, which has led to a diversity of generators available in computer software, public domain and so on. For many of these algorithms (and their implementations) only a few rudimentary tests have been performed.

In this work we have undertaken an extensive test program [58] of a group of pseudorandom number generators, which are often employed in the applications of physics. These generators which are described in detail in Section 2 include public domain programs GGL, RANMAR, RAN3, RCARRY and R250, a library subroutine G05FAF, manufacturer installed routines RAND and RANF, and even a sequence generated from physical noise (PURAN II). Our strategy is to perform a large set of different tests for all of these generators, whose results can then be directly compared with each other. There are two main reasons for this. Namely, there is a difficulty associated with most quantitative tests in the choice of the test parameters and final criteria for judging the results. Thus, we think that full comparative tests of a large group of generators using identical test parameters and criteria can yield more meaningful results, in particular when there is a need for a reliable generator with a good overall performance. Second, performing a large number of tests also allows a comparison of exactly how efficient each test is in finding certain kinds of correlations.

As discussed in Section 3, we first employ an array of standard statistical tests, which measure the degree of uniformity of the distribution of numbers, as well as correlations between them. Following this, we perform a series of bit level tests, some of which should be particularly efficient in finding correlations between consecutive bits in the random number sequences. Third part of our testing utilizes visual pictures of random numbers and their bits on a plane. Finally, for the sake of completeness we have also included a relative performance test of the generators in our results. A complete summary of the test results is presented in Section 4. As our main result we find three generators, namely GGL, G05FAF, and R250 with an overall best performance in all our tests, although some other generators such as RANF and RANMAR perform almost as convincingly. We also find that the bit level tests are most efficient in finding local correlations in the random 
numbers, but do not nevertheless guarantee good statistical properties, as shown in the case of RCARRY. Our results also show that visual tests can indeed reveal spatial correlations not clearly detected in the quantitative tests. Our work thus provides a rather comprehensive test bench which can be utilized in choosing a random number generator for a given application. However, choosing a "good quality" random number generator for all applications may not be trivial as discussed in Section 5 , in light of the recent results reporting anomalous correlations in Monte Carlo simulations [14, 23] using the here almost impeccably performing R250. Thus, more physical ways of testing random number sequences are probably needed, a project which is currently underway [59]. 


\section{Generation of Random Numbers}

"The generation of random numbers is too important to be left to chance."

R. Coveyou

Pseudorandom number sequences needed for high speed applications are usually generated at run time using an algorithm which often is a relatively simple nonlinear deterministic map. The implementation of the corresponding recurrence relation must also ensure that the stream of numbers is reproducible from identical initial conditions. The deterministic nature of generation means that the designer has to be careful in the choice of the precise relationship of the recursion, otherwise unwanted correlations will appear as amply demonstrated in the literature [8].

However, even the best generator algorithm can be defeated by a poor computer implementation. Whenever an exact mathematical algorithm is translated into a computer subroutine, different possibilities for its implementation may exist. Only if the operation of a generator can be exactly specified on the binary level, has the implementation a chance to be unambiguous; otherwise, machine dependent features become incorporated into the routine. These include finite precision of real numbers, limited word size of the computer, and numerical accuracy of mathematical functions. Furthermore, it would often be desirable that the implemented routine performed identically in each environment in which it is to be executed, i.e. it would be portable.

Some of the desired properties of good pseudorandom number generators are easily defined but often difficult to achieve simultaneously. Namely, besides good "randomness" properties portability, repeatability, performance speed, and a very long period are often required. Ideally, a random number generator would be designed for each application, and then tested within that application to ensure that the inevitable correlations that do exist in a deterministic algorithm, cause no observable effects. In practice, this is seldom possible, which is another reason why extensive tests of pseudorandom number generators are needed.

Most commonly used pseudorandom number generator algorithms are the linear congruential method, the lagged Fibonacci method, the shift register method, and combination methods. A special case are nonalgorithmic or physical generators which are used for creating a non - reproducible sequence of random numbers. These are usually based on "random" physical events, e.g. changes in physical characteristics 
of devices, cosmic ray bursts or electromagnetic interference. Details and properties of the algorithms will be summarized in the next section. Following this, we shall describe in more detail the particular generators chosen for our tests. Reviews of current state of generation methods can be found in e.g. Marsaglia [42, James [27, L'Ecuyer [34], and Anderson [3].

\subsection{Classification of Generation Methods}

"Anyone who considers arithmethical methods of producing random digits is, of course, in a state of sin."

J. VON NEUMANN

Among the simplest algorithms are the linear congruential generators which use the integer recursion

$$
X_{i+1}=\left(a X_{i}+b\right) \bmod m,
$$

where the integers $a, b$ and $m$ are constants. It generates a sequence $X_{1}, X_{2}, \ldots$ of random integers between 0 and $m-1$ (or in the case $b=0$, between 1 and $m-1$ ). Each $X_{i}$ is then scaled into the interval $[0,1)$. Parameter $m$ is often chosen to be equal or nearly equal to the largest integer in the computer. Linear congruential generators can be classified into mixed $(b>0)$ and multiplicitive $(b=0)$ types, and are usually denoted by $\operatorname{LCG}(a, b, m)$ and $\operatorname{MLCG}(a, m)$, respectively.

Since the introduction of this algorithm by Lehmer [35], its properties have been researched in detail. Marsaglia 40] pointed out about 20 years ago that the random numbers in $d$ dimensions lie on a relatively small number of parallel hyperplanes. Further theoretical work [11, 15, 16] has been done to weed out bad choices of the constants $a, b$ and $m$ but so far no consensus has evolved on a unique best choice for these parameters.

To increase the period of the linear congruential algorithm, it is natural to generalize it to the form

$$
X_{i}=\left(a_{1} X_{i-1}+\cdots+a_{p} X_{i-p}\right) \bmod m,
$$

where $p>1$ and $a_{p} \neq 0$. The period is the smallest positive integer $\lambda$ for which

$$
\left(X_{0}, \ldots, X_{p-1}\right)=\left(X_{\lambda}, \ldots, X_{\lambda+p-1}\right) .
$$

Since there are $m^{p}$ possible $p$ - tuples, the maximum period is $m^{p}-1$. In this category the simplest algorithm is of the Fibonacci type. The use of $p=2, a_{1}=$ 
$a_{2}=1$ leads to the Fibonacci generator

$$
X_{i}=\left(X_{i-1}+X_{i-2}\right) \bmod m
$$

Since no multiplications are involved, this implementation has the advantage of being fast.

A lagged Fibonacci generator requires an initial set of elements $X_{1}, X_{2}, \ldots, X_{r}$ and then uses the integer recursion

$$
X_{i}=X_{i-r} \otimes X_{i-s}
$$

where $r$ and $s$ are two integer lags satisfying $r>s$ and $\otimes$ is a binary operation $(+,-$, $\times, \oplus$ (exclusive-or $))$. The corresponding generators are designated by $\operatorname{LF}(r, s, \otimes)$. Typically, the initial elements are chosen as integers and the binary operation is addition modulo $2^{n}$. Lagged Fibonacci generators are elaborated in e.g. Ref. 44.

An alternative generator type is the shift register generator. Feedback shift register generators are also sometimes called Tausworthe generators [54. The feedback shift register algorithm is based on the theory of primitive trinomials of the form $x^{p}+x^{q}+1$. Given such a primitive trinomial and $p$ binary digits $x_{0}, x_{1}, x_{2}, \ldots, x_{p-1}$, a binary shift register sequence can be generated by the following recurrence relation:

$$
x_{k}=x_{k-p} \oplus x_{k-p+q},
$$

where $\oplus$ is the exclusive-or operator, which is equivalent to addition modulo 2 . $l$-bit vectors can be formed from bits taken from this binary sequence as

$$
W_{k}=x_{k} x_{k+d} x_{k+2 d} \cdots x_{k+(l-1) d},
$$

where $d$ is a chosen delay between elements of this binary vector. The resulting binary vectors are then treated as random numbers. Such a generated sequence of random integers will have the maximum possible period of $2^{p}-1$, if $x^{p}+x^{q}+1$ is a primitive trinomial and if this trinomial divides $x^{n}-1$ for $n=2^{p}-1$, but for no smaller $n$. These conditions can easily be met by choosing $p$ to be a Mersenne prime, i.e. a prime number $p$ for which $2^{p}-1$ is also a prime. A list of Mersenne primes can be found e.g. in Refs. 60, 62, 6, 25]. Generators based on small values of $p$ do not perform well on the tests [42]. According to some statistical tests on computers [57] the value of $q$ should be small or close to $p / 2$.

Lewis and Payne 37 formed $l$-bit words by introducing a delay between the words. The corresponding generator is called the generalized feedback shift register generator, denoted by $\operatorname{GFSR}(p, q, \oplus)$. In a GFSR generator the words $W_{k}$ satisfy the 
recurrence relation:

$$
W_{k}=W_{k-p} \oplus W_{k-p+q} .
$$

Under special conditions, maximal period length of $2^{p}-1$ can be achieved. Lewis and Payne [37] and Niederreiter [47] have also studied the properties of the algorithm theoretically. An important aspect of the GFSR algorithm concerns its initialization, where $p$ initial seeds are required. This question has been studied theoretically in Refs. [18, 19, 55, 56, 20].

Given the inevitable dependencies that will exist in a pseudorandom sequence, it seems natural that one should try to shuffle a sequence [26] or to combine separate sequences. An example of such approach is given by MacLaren and Marsaglia [39] who were apparently the first to suggest the idea of combining two generators together to produce a single sequence of random numbers. The essential idea is that if $X_{1}, X_{2}, \ldots$ and $Y_{1}, Y_{2}, \ldots$ are two random number sequences, then the sequence $Z_{1}, Z_{2}, \ldots$ defined by $Z_{i}=X_{i} \otimes Y_{i}$ will not only be more uniform than either of the two sequences but will also be more independent. Algorithms using this idea are often called mixed or combination generators.

As mentioned before, physical devices have also been used in the creation of random number sequences. Usually, however, such sequences are generated too slowly to be used in real time, but rather stored in the computer memory where they can be easily accessed. This also guarantees the reproducability of the chosen sequence in applications. However, physical memory restrictions often severely limit the number of stored numbers. Unwanted and unknown physical correlations may also affect the quality of physical random numbers. As a result, physical random numbers have not been commonly used in simulations. One implementation of a physical generator can be found in Ref. [52].

\subsection{Descriptions of Generators}

In this section, we shall describe in more detail the generators which have been chosen for the tests. Since many combinations of possible parameters exist, we have tried to choose those particular algorithms which have been most commonly used in physics applications, or which have been previously tested. At the end of this section, we shall also describe a sequence of random numbers generated from 
physical noise, which has been included for purposes of comparison.

\section{- GGL}

GGL is a uniform random number generator based on the linear congruential method [48]. The form of the generator is MLCG $\left(16807,2^{31}-1\right)$ or

$$
X_{i+1}=\left(16807 X_{i}\right) \bmod \left(2^{31}-1\right) .
$$

This generator has been particularly popular [48]. It has seen extensive use in the IBM computers [65], and is also available in some commercial software packages such as subroutine RNUN in the IMSL library [66] and subroutine RAND in the MATLAB software [67]. MLCG $\left(16807,2^{31}-1\right)$ generators are quite fast and have been argued to have good statistical properties [36]. Results of tests with and without shuffling are reported by Learmonth and Lewis [32]. Other test results on implementations of this algorithm have been given in [29, 2, 3, 34]. Its drawback is its cycle length $2^{31}-1\left(\approx 2 \times 10^{9}\right.$ steps $)$ [29], which can be exhausted fast on a modern high speed computer. We also note that our Fortran implementation of GGL is particularly sensitive to the arithmetic accuracy of its implementation (cf. Section 4). Our Fortran implementation of GGL produces the same sequence as RNUN of the IMSL library 1

\section{- RAND}

RAND uses a linear congruential random number algorithm with a period of $2^{32}$ [63] to return successive pseudorandom numbers in the range from 0 to $2^{31}-1$. The generator is $\operatorname{LCG}\left(69069,1,2^{32}\right)$ or

$$
X_{i+1}=\left(69069 X_{i}+1\right) \bmod 2^{32} .
$$

The multiplier 69069 has been used in many generators, probably because it was strongly recommended in 1972 by Marsaglia [41], and is part of the SUPER - DUPER generator [3]. Test results on various implementations of the LCG $\left(69069,1,2^{32}\right)$ algorithm have been reported in [32, 42, 3, 44]. The generator tested here is the implementation by Convex Corp. on the Convex C3840 computer system [63].

\section{- RANF}

\footnotetext{
${ }^{1}$ We also unsuccessfully tried the IBM assembly code implementation of Lewis et al. [36] on an IBM 3090 computer.
} 
The RANF algorithm uses two equations for generation of uniform random numbers. It utilizes the multiplicative congruential method with modulus $2^{48}$. The algorithms are $\operatorname{MLCG}\left(M_{1}, 2^{48}\right)$ and $\operatorname{MLCG}\left(M_{64}, 2^{48}\right)$ :

$$
\begin{gathered}
X_{i+1}=\left(M_{1} X_{i}\right) \bmod 2^{48}, \\
X_{i+64}=\left(M_{64} X_{i}\right) \bmod 2^{48},
\end{gathered}
$$

where $M_{1}=44485709377909$ and $M_{64}=247908122798849$. Period length of the RANF generator is $2^{46}$ [45]. Spectral test results on the RANF generator have been given in Refs. [3, 17]. On the CRAY-X/MP and CRAY-Y/MP systems, RANF is a standard vectorized library function 64]. The operations $\left(M_{1} X_{i}\right)$ and $\left(M_{64} X_{i}\right)$ are done as integer multiplications in such a way as to preserve the lower 48 bits. We tested RANF on a Cray X-MP/432.

\section{- G05FAF}

G05FAF is a library routine in the NAG software package 68. It calls G05CAF which is a multiplicative congruential algorithm $\operatorname{MLCG}\left(13^{13}, 2^{59}\right)$ or

$$
X_{i+1}=\left(13^{13} X_{i}\right) \bmod 2^{59} .
$$

G05FAF can be used to generate a vector of $n$ pseudorandom numbers which are exactly the same as $n$ successive calls to the G05CAF routine. Generated pseudorandom numbers are uniformly distributed over the specified interval $[a, b)$. The period of the basic generator is $2^{57}$ [68]. Its performance has been analyzed by the spectral test [30].

\section{- R250}

R250 is an implementation of a generalized feedback shift register generator [37]. The 31-bit integers are generated by a recurrence of the form $\operatorname{GFSR}(250,103, \oplus)$ or

$$
X_{i}=X_{i-250} \oplus X_{i-(250-103)} .
$$

Implementation of the algorithm is straightforward, and $p=250$ words of memory are needed to store the 250 latest random numbers. A new term of the sequence can be generated by a simple exclusive - or operation. An IBM assembly language implementation of this generator has been presented by Kirkpatrick and Stoll 229 who use a MLCG $\left(16807,2^{31}-1\right)$ to produce the first 250 initializing integers. Due to the popularity of R250, there have been many different approaches for its initialization 
[50, 18, 7, 20]. The period of the generator is $2^{250}-1$ [29]. Some test results of R250 generator have been reported by Kirkpatrick and Stoll [29]. We have implemented R250 on Fortran [24.

\section{- RAN3}

RAN3 generator is a lagged Fibonacci generator $\operatorname{LF}(55,24,-)$ or

$$
X_{i}=X_{i-55}-X_{i-24}
$$

The algorithm has also been called a subtractive method. The period length of RAN3 is $2^{55}-1$ [30, and it requires an initializing sequence of 55 numbers. The generator was originally Knuth's suggestion [30] for a portable routine but with an add operation instead of a subtraction. This was translated to a real Fortran implementation by Press et al. [51]. We were unable to find any published test results for RAN3.

\section{- RANMAR}

RANMAR is a combination of two different generators [27, 43]. The first is a lagged Fibonacci generator

$$
X_{i}= \begin{cases}X_{i-97}-X_{i-33}, & \text { if } X_{i-97} \geq X_{i-33} \\ X_{i-97}-X_{i-33}+1, & \text { otherwise }\end{cases}
$$

Only 24 most significant bits are used for single precision reals. The second part of the generator is a simple arithmetic sequence for the prime modulus $2^{24}-3=$ 16777213. The sequence is defined as

$$
Y_{i}= \begin{cases}Y_{i}-c, & \text { if } Y_{i} \geq c \\ Y_{i}-c+d, & \text { otherwise }\end{cases}
$$

where $c=7654321 / 16777216$ and $d=16777213 / 16777216$.

The final random number $Z_{i}$ is then produced by combining the obtained $X_{i}$ and $Y_{i}$ as

$$
Z_{i}= \begin{cases}X_{i}-Y_{i}, & \text { if } X_{i} \geq Y_{i} \\ X_{i}-Y_{i}+1, & \text { otherwise }\end{cases}
$$


The total period of RANMAR is about $2^{144}$ [43]. A scalar version of the algorithm has been tested on bit level with good results 43. We used the implementation by James [27] which is available in the Computer Physics Communications (CPC) software library, and has been recommended for a universal generator.

\section{- RCARRY}

RCARRY 44] is based on the operation known as "subtract - and - borrow". The algorithm is similar to that of lagged Fibonacci, but it has the occasional addition of an extra bit. The extra bit is added if the Fibonacci sum is greater than one. The basic formula is:

$$
X_{i}=\left(X_{i-24} \pm X_{i-10} \pm c\right) \bmod b .
$$

The carry bit $c$ is zero if the sum is less than or equal to $b$, and otherwise " $c=1$ in the least significant bit position" [27]. The choice for $b$ is $2^{24}$.

The period of the generator is about $2^{1407}$ 44 when 24-bit integers are used for the random numbers. We were unable to find any published test results for RCARRY. We used the implementation of James [27], again available in the CPC software library.

\section{- PURAN II}

PURAN II is a physical random number generator created by Richter [52]. It uses random noise from a semiconductor device. The generated data has been permanently stored on a computer disk, from which it can be transferred by request. In this work, we have tested the PURAN II data on bit level only (cf. Section 4), and also used it to verify the correct operation of our test programs. 


\section{Description of the Tests}

"Of course, the quality of a generator can never be proven by any statistical test."

P. L'ECUYER

A fundamental problem in testing finite pseudorandom number sequences stems from the fact that the definition of randomness for such sequences is not unique [31]. Thus, one usually has to decide upon some criteria which test at least the most fundamental properties that such sequences should possess, such as correct values of the moments of their probability distribution. This has lead to the emergence of a large number of tests which can be divided into three approximate categories: Statistical (or traditional) tests for testing random numbers in real or integer representation, bit level tests for binary representations of random numbers, and more phenomenological visual tests. In this work, we have employed several tests belonging to each of these categories, as will be discussed below. Also, the spectral test for LCG generators was included. We should note here that recently Compagner and Hoogland [8] have suggested a more systematic test program for finite sequences. We shall not employ it in this work, however.

The traditional utilitarian approach has been to subject pseudorandom number sequences to tests, which derive from mathematical statistics [30. In their simplest form, tests in this category reveal possible deviations of the distribution of numbers from an uniform distribution, such as the $\chi^{2}$ test. However, some of the more sophisticated tests should actually probe correlations between successive numbers as well [33.

Another approach is to test the properties of random numbers on the bit level. Of the traditional tests, some can be performed in this manner also. Marsaglia [42] has proposed additional tests which explicitly probe the individual bits of random number sequences represented as binary computer words. Some of these tests have been further refined [2]. We have included two of these tests here, in particular to examine possible correlations between bits of successive binary words.

A rather different way of testing spatial correlations between random numbers is possible by using direct visualization. This can most easily be done in two dimensions by plotting pairs of points on a plane, or visualizing the bits of binary numbers. In addition to yielding qualitative information, such tests offer a possibility to develop more physical quantitative tests through interpretation of the visualized configu- 
rations as representations of physical systems, such as the Ising model [8, 59]. In this work, however, we have simply used a few different types of visual tests to complement our quantitative tests.

Before discussing each test in detail, we would like to emphasize that although some of the generators we have tested have previously been subjected to similar tests, an extensive comparative testing of a large collection of generators has been lacking up to date. The importance of this becomes obvious when one considers the freedom of choice of various parameters in the tests, as discussed below. Only comparative testing with identical parameters allows a direct comparison between different generators. Another difficulty concerns the implementation of random number generator algorithms and the testing routines [53, 48, 3, 22]. Problems in either may actually lead to significant differences in the results. In fact, as an example we shall explicitly demonstrate for GGL and RAND how slightly different implementations of the same generator can lead to completely different results.

\subsection{Statistical tests}

The statistical tests included in our test bench were the uniformity test, the serial test, the gap test, the maximum of $t$ test, the collision test, and the run test. In addition, we carried out the park test [42]. A review of the statistical tests can be found e.g. in Ref. [30, and a suggestion for implementing them in Ref. [13].

The leading idea in carrying out the statistical tests was to improve the statistical accuracy of these tests by utilizing a one way Kolmogorov - Smirnov (KS) test. This was achieved by repeating each individual test described below $N$ times, and then submitting the obtained empirical distribution to a KS test (for the park test, however, this was not possible). Similar approach has been suggested earlier by Dudewicz and Ralley [13] and realized by L'Ecuyer [33]. The KS test reveals deviations of an empirical distribution function $\left(F_{n}(x)\right)$ from the theoretical one $(F(x))$. This can be quantified by test variables $K^{+}$and $K^{-}$, which are defined by $K^{+}=\sqrt{n} \sup \left\{F_{n}(x)-F(x)\right\}$ and $K^{-}=\sqrt{n} \sup \left\{F(x)-F_{n}(x)\right\} . K^{+}$measures the maximum deviation of $F_{n}(x)$ from $F(x)$ when $F_{n}(x)>F(x)$ and $K^{-}$measures the respective quantity for $F_{n}(x)<F(x)$. The tests are as follows:

(i) To test the uniformity of a random number sequence, a standard $\chi^{2}$ test was used [30]. $n$ random numbers were generated in the half open interval $[0,1)$, then multiplied by $\nu$ and truncated to integers in the interval $[0, \nu)$. The 
number of occurrences in each of the $\nu$ bins was compared to the theoretical prediction using the $\chi^{2}$ test.

(ii) Serial correlations were tested [32, 30] by studying the occurrence of $d$-tuples of $n$ random numbers distributed in the interval [0,1). For example, in the case of pairs, we tabulated the number of occurrences of $\left(x_{2 i}, x_{2 i+1}\right)$ for all $i \in$ $[0, n)$. Each $d$-tuple occurs with the probability $\nu^{-d}$ where $\nu$ is the number of bins in the interval. The results were then subjected to the $\chi^{2}$ test.

(iii) The gap test 30 probes the uniformity of the random number sequence of length $n$. Once a random number $x_{i}$ falls within a given interval $[\alpha, \beta]$, we observe the number of subsequent numbers $x_{i+1} \ldots x_{i+j-1} \notin[\alpha, \beta]$. When again $x_{j} \in[\alpha, \beta]$, it defines a gap of length $j$. For finite sequences, it is useful to define a maximum gap length $l$. Then we can test the results against the theoretical probability using the $\chi^{2}$ test.

(iv) The maximum of $t$ test [30] is a simple uniformity test. If we take a random number sequence of length $n\left(x_{i} \in[0,1), i=1, \ldots, n\right)$ and divide it into subsequences of length $t$ and pick the maximum value for each subsequence, the maxima should follow the $x^{t}$ distribution.

(v) The collision test 30 can be used to test the uniformity of the sequence when the number of available random numbers $(n)$ is much less than the number of bins $(w)$. We then study how many times a random number falls in the same bin, i.e. how many collisions occur. The probability for $j$ collisions is:

$$
\frac{w(w-1) \cdots(w-n+j+1)}{w^{n}}\left(\begin{array}{c}
n \\
n-j
\end{array}\right),
$$

where $w=s^{d}, d$ is the dimension and $s$ can be chosen.

(vi) In the run test 32, 30, we calculate the number of occurrences of increasing or decreasing subsequences of length $1 \leq i<l$ for a given random number sequence $x_{1}, x_{2}, \ldots, x_{n}$. To carry out this test we chose $l=6$ and followed Knuth [30] in the choice of the relevant test quantity.

(vii) In the park test 42, we choose randomly points in a $d$-dimensional space and allocate a diameter for each point. Within each diameter, "a car is parked". The aim is to park as many non - overlapping cars as possible, and study the distribution of $k$ cars. Unfortunately, since the theoretical distribution is not known, this test can only be used for qualitative comparative studies. 


\subsection{Bit level tests}

Two of the tests included in the previous section on statistical tests, namely the run test and the collision test could equally well be included in the category of bit level tests as they can also be performed for binary representations of random numbers. Recently, Marsaglia 42 has introduced new tests in his DIEHARD random number generator test bench. Of these we carried out the $d$-tuple and the rank tests. We shall briefly describe both of them below.

(i) The $d$-tuple test realized here is a modified version [2] of the original [42]. We extended the test by improving its statistical accuracy by submitting the empirically obtained distribution to a Kolmogorov - Smirnov test. In the $d$ tuple test, we represent a random integer $I_{i}$ as a binary sequence of $s$ bits $b_{i, j},(j=1, \ldots, s)$ :

$$
\begin{aligned}
I_{1}= & b_{1,1} b_{1,2} b_{1,3} \cdots b_{1, s}, \\
I_{2}= & b_{2,1} b_{2,2} b_{2,3} \cdots b_{2, s}, \\
& \vdots \\
I_{n}= & b_{n, 1} b_{n, 2} b_{n, 3} \cdots b_{n, s},
\end{aligned}
$$

where an obvious choice for the parameter $s=31$ (in testing RAN3, we used $s=30)$. Each of the binary sequences $I_{i}$ is divided into subsequences of length $l$ which can be used to form $n$ new binary sequences $I_{i}^{\prime}=b_{i, 1} b_{i, 2} \ldots b_{i, l}$. These sequences are then joined into one more binary sequence of length $d \times l$ in such a way that these final sequences $\bar{I}_{i}$ partially overlap:

$$
\begin{aligned}
\bar{I}_{1}= & b_{1,1} \cdots b_{1, l} b_{2,1} \cdots b_{2, l} \cdots b_{d, 1} \cdots b_{d, l}, \\
\bar{I}_{2}= & b_{2,1} \cdots b_{2, l} b_{3,1} \cdots b_{3, l} \cdots b_{d+1,1} \cdots b_{d+1, l}, \\
& \vdots \\
\bar{I}_{n}= & b_{n, 1} \cdots b_{n, l} b_{n+1,1} \cdots b_{n+1, l} \cdots b_{n+d-1,1} \cdots b_{n+d-1, l} .
\end{aligned}
$$

Each of these new integers falls within $\bar{I}_{i} \in\left[0,2^{d l}-1\right]$. In the test, the values $\bar{I}_{i}$ of the new random numbers are calculated as well as the number of respective occurrences. A statistic which follows the $\chi^{2}$ distribution can be calculated although the subsequent sequences are correlated [2]. The $N$ results of the $\chi^{2}$ test were finally subjected to a KS test.

(ii) For the rank test, we construct a $(v \times w)$ random binary matrix from the random numbers. The probability that the rank $r$ of such a matrix equals 
$r=0,1,2, \ldots, \min (w, v)$ can be calculated 42 allowing us to perform the $\chi^{2}$ test, followed by a KS test.

\subsection{Spectral test}

The spectral test was included for the sake of completeness. Unlike other tests, it relies on the theoretical properties of LCG algorithms independent of their implementation. It has been used extensively to characterize the properties of linear congruential generators [11, 30, 15, 16, 33, 48, 3, 17, mainly in order to find "good" values for the parameters within them. It probes the maximal distance between hyperplanes on which the random numbers produced by an LCG generator fall 40. The smaller the distance, the "better" the generator.

All the linear congruential generators used in this study were subjected to the spectral test. We used two figures of merit [30], namely

$$
\kappa_{d}=\frac{\nu_{d}}{\gamma_{d} m^{\frac{1}{d}}}
$$

where $d$ is dimensionality and $m$ is the period of the LCG generator in question. The wave number $\nu_{d}$ is the inverse of the maximal distance between the hyperplanes in $d$ dimensions, and the coefficient $\gamma_{d}$ depends on dimension and is tabulated e.g. in Knuth [30]. Basically, the denominator is the inverse of the theoretical minimal distance between hyperplanes [33], and thus $\kappa_{d}$ is the normalized distance between hyperplanes. The other figure of merit is

$$
\lambda_{d}=\log _{2}\left(\nu_{d}\right)
$$

which gives the number of bits uniformly distributed in $d$ dimensions [30, 3]. The larger $\lambda_{d}$, the "better" the generator.

\subsection{Visual tests}

Visual tests can provide additional qualitative information about the properties of random number generators and can further corroborate the results of quantitative tests. We submitted the generators to four visual tests:

(i) The distribution of random number pairs was plotted in two dimensions to see if there exists any ordered structures. For an LCG generator, one should be 
able to distinguish the hyperplanes on which the random numbers fall [40, 30]. The shorter the interplanar distance, the "better" the generator. Also lagged Fibonacci generators as well as shift register ones are known to produce some structure which should be visible with some choices of parameters [8, 3].

(ii) To study binary sequences visually one can plot the random numbers as binary computer words on a plane. Ones were mapped onto black squares and zeros onto white ones. The consequent figure can also be interpreted as a configuration of a two dimensional Ising model at an infinite temperature which could be subjected to quantitative tests [59].

(iii) First $n$ random numbers were generated. Then the distance $\left|x_{i}-x_{j}\right|$ from $x_{i}$ was calculated for all $j=1, \ldots, n$. This was done for all $x_{i}(i=1, \ldots, n)$, and then the distance was plotted in two dimensions with gray scale colors: the lighter the color, the larger the difference. Areas of uniform gray shade indicate possible local correlations in the random number sequence.

(iv) The gap test was visualized by calculating the difference $\left|x_{i}-x_{j}\right|$, where $i \in$ $[\alpha, \beta]$ and $j=1, \ldots, l$ where $l$ is the maximum gap length as in section 3.1. The difference was plotted in gray shade colors as in (iii) above. Uniform darkening or lightening of gray shades indicates a gap. 


\section{Results}

"A random number generator is much like sex: when it's good it's wonderful, and when it's bad it's still pretty good."

G. Marsaglia

The random number generators were initialized with the seed 667790 $\left(=10100011000010001110_{2}\right)$ except for R250 which was initialized with GGL (to 24 significant bits) using this seed. Following initialization, consequtively generated sequences of random numbers were subjected to statistical and bit level tests which were repeated $N$ times, after which the one way Kolmogorov - Smirnov test was applied to the $N$ results to further improve the statistics. Thus, the final test variables are the $K^{-}$and $K^{+}$values. The only exception is the maximum of $t$ test where an additional KS test was applied to the results of the first KS test. A generator was considered to fail a test if the descriptive level $\delta^{-}$or $\delta^{+}$ was less than 0.05 33 or larger than 0.95. In other words, a failure occurred if the empirical distribution followed too closely or was too far from the theoretical one. We note that to verify the independence of the results on the choice of the seed, two other choices for initial seeds for RAND and RAN3 were tested namely 1415926535 (= $1010100011001010101001100000111_{2}$ ) (from the decimals of $\pi$ ), and $2^{15}\left(=32768=1000000000000000_{2}\right)$. No changes in the results of the $d$-tuple test were found. Our tests were performed on a Convex C3840, a Silicon Graphics Iris 4D380 VGX and a Kubota Titan 3000. A Cray X-MP was used for testing RANF.

\subsection{Standard tests}

The parameters used in the standard tests are given in Table 1. The numbering refers to Table 2 where the results are shown. In the choice of parameters, we followed L'Ecuyer [33], with some changes to improve the statistical accuracy of the results. If a generator failed a given test, it was subjected to another test starting from the state it reached in the first test. If a second failure occurred, one more test was performed by starting from a new initial state with the seed 14159 $\left(=11011101001111_{2}\right)$.

In Table 2, frames with thin lines indicate a single failure, frames with double single lines two failures, and frames with bold lines three consequtive failures in the corresponding tests. Additionally, as overall "goodness" factors for each generator, we 
have calculated relative deviations $R_{\delta}$ and $R_{K}$ from the average theoretical descriptive level values $\delta^{+}$and $\delta^{-}$(shown in the table) and the corresponding KS test values $K^{+}$and $K^{-}$, respectively. They are shown at the bottom of the table. Based on our results, the performance of the generators falls in three rough categories. GGL, R250, RANF, and G05FAF all display only single failures, RAND and RAN3 fail two consecutive times, and RANMAR and RCARRY fail there consequtive times at least once. The calculated goodness values support these results, too. The performance of RCARRY is noticeably poor in the gap test which suggests possible local correlations in the random number sequence. We should note that although the calculated $R$ values give an indication of the overall performance, it is clear that one should be aware of the particular weaknesses of each generator before a specific application is considered. Finally, as a qualitative counterpart to the statistical tests, the park test was carried out with two different "car sizes", i.e. $d=0.001, n=10^{6}$, and $d=0.01, n=10^{5}$. However, we found the results for all generators to agree within errors. Thus, the test gave no additional information in the present case.

When comparing our results with the literature, one should note that the actual implementations of the generators tested may differ from ours. Different implementations of the same algorithm may change the generated random number sequence, with unknown consequences for its properties. This makes direct comparisons of the results difficult. Another problem lies in the possible machine dependence of a "bad" implementation. An example of this is GGL, which when implemented in single precision mode in 32 bit computers gives a period of 32 , as will be discussed later in the context of the visual test results.

Various implementations of the algorithm MLCG(16807, $\left.2^{31}-1\right)$ (GGL here) have previously been tested extensively, see e.g. Refs. [36, 32, 21, 29]. In particular, Lewis et al. [36] performed a series of tests when they introduced the IBM SYSTEM/360 assembly language implementation of MLCG $\left(16807,2^{31}-1\right)$. They used sequences of lengths $n=2^{16}+5=65541$ with tests repeated ten times. The authors characterized the quality of the results by the maximum standard deviations $\sigma_{\max }$ from the mean. In the uniformity test, the sequence was divided into $\nu=2^{12}=4096$ bins, and all results were within $\sigma_{\max }=1.9$ from the mean. The serial test for pairs $\left(x_{i}, x_{i+l}\right)$ was performed with various lags $l$ and number of bins $\nu=256$, with results within $\sigma_{\max }=$ 2.3. Furthermore, the run test was completed with $l=8, n=65541$, and repeated ten times, with "much larger" standard deviations. However, Lewis et al. concluded that the run test together with their other tests gave "no evidence of departures from randomness" [36]. In another reference [32], an unspecified implementation of the 
generator has also been subjected to the run test with a two way KS test and has been found to pass it, as well as a serial test for triples $(d=3)$ but to fail a serial test for pairs $(d=2)$. In the run test, the sequence length was $n=65536$, the runs were counted up to $l=8$ and the test was repeated one hundred times $(N=100)$. Similarly, in the serial test $n=65541$ and $N=100$ [32]. We should note that in our tests, where better statistics was used $(N=1000)$ GGL passed the run test and all the serial tests for $d=2,3,4$.

RAND is an implementation of $\operatorname{LCG}\left(69069,1,2^{32}\right)$ by Convex Corp. [63]. Learmonth and Lewis [32] have also tested their assembly language implementation of $\operatorname{LCG}\left(69069,1,2^{32}\right)$ with the same test as the $\operatorname{MLCG}\left(16807,2^{31}-1\right)$ generator discussed above. $\operatorname{LCG}\left(69069,1,2^{32}\right)$ passed the serial test for both $d=2$ and $d=3$, as well as the run test. In our tests, RAND passed all these tests as well.

An IBM assembly language implementation of $\operatorname{GFSR}(250,103, \oplus)$ has been found to perform well in a run test with parameters $n=10^{6}, N=30, l=9$ [29. Our Fortran implementation of it, namely R250 also passed the run test.

Finally, we have been unable to find any published data on statistical tests for any implementations of RANMAR, RCARRY, RAN3, RANF or G05FAF.

\subsection{Spectral test}

The results of the spectral test for the LCG generators of this paper are presented in Table 3. In the case of RANF, we show results for the two generators which comprise it, namely MLCG(44485709377909, $2^{48}$ ) (RANF1) and MLCG(247908122798849,248) (RANF2). Overall, the generator G05FAF from the NAG library is the most successful in the test. On the other hand, GGL displays the known flaw of this generator performing poorly at low dimensions. All generators, however, gave worse results in most dimensions than the minimum acceptable values suggested by Fishman and Moore [16, 3]. We note that since the results of this test are independent of the implementations of the algorithms, our results for RAND agree with previous results for $\operatorname{LCG}\left(69069,1,2^{32}\right)$ [3], for GGL with $\operatorname{MLCG}\left(16807,2^{31}-1\right)$ [16, 33, 3] and for RANF1 with MLCG(44485709377909, $\left.2^{48}\right)$ [3, 17]. 


\subsection{Bit level tests}

The bit level tests probe the properties of the individual bits which comprise the random numbers thus testing properties somewhat different from the statistical tests. We chose to use the generalized $d$-tuple test and the rank of a random matrix test for studying correlations on the bit level.

The $d$-tuple test $(d=3)$ was carried out for $n=5000$ random numbers, each of which was coded into a 31 bit binary sequence (for RAN3, the sequence length was 30). Of this sequence, we chose bit strips of width $l=3$. The $\chi^{2}$ test was performed $N=1000$ times, and the results were then subjected to a KS test2. This test was performed twice for each generator (excluding PURAN II) and we considered "failed" only those bits that failed twice in succession.

The rank test was carried out with parameters $v=w=2, n=1000$ and $N=1000$. The $(2 \times 2)$ random matrices were formed systematically using the $i^{\text {th }}$ and $(i+1)^{\text {th }}$ $(1 \leq i \leq 31)$ bit pairs from each two successive numbers. The test was performed twice to all pseudorandom number generators with the same failing criteria as in the $d$-tuple test.

Results for the $d$-tuple and rank tests are shown in Table 4 . Details of the implementations and initializations of the generators are also shown there. In our notation, the bit number one is the most significant bit. GGL, G05FAF, and R250 pass both tests with an impeccable performance, in that none of the 31 bits show observable correlations. The physically generated random numbers of PURAN II also pass both tests. For RANMAR and RCARRY, only the 24 most significant bits are guaranteed to be good [27], which our tests confirm. On the other hand, RAND and RAN3 show significant correlations. In particular, the correlations in RAN3 are serious since they affect the five most significant bits. When RAND was called in integer form, it gave one more correlated bit than the calls in floating point representation both in the $d$-tuple and rank tests.

Previously, an unspecified implementation of $\operatorname{MLCG}\left(16807,2^{31}-1\right)$ (GGL here) has been shown to pass a simpler version of the $d$-tuple test $(d=l=3, n=2000$, test repeated five times) [2]. An unspecified implementation of LCG(69069, 1, $\left.2^{32}\right)$ (RAND here) failed the same test with 11 failing bits [2]. An IBM assembly language implementation of $\operatorname{GFSR}(250,103, \oplus)$ (R250 here) and an unspecified implementation

\footnotetext{
${ }^{2}$ Results of a systematic study indicate [59] that with these parameters, the $d$-tuple test can detect correlations up to about fifty numbers apart.
} 
of MLCG(16807, $\left.2^{31}-1\right)$ (GGL here) have been shown to pass a test which probed possible correlations in the five most significant bits by studying triples of random numbers by placing them on a unit cube with a resolution of $32 \times 32 \times 32$ cells ( $n=10^{6}$ and the test was repeated "several" times) [29]. Kirkpatrick and Stoll [29] further argue that as all columns of bits generated by a $\operatorname{GFSR}(250,103, \oplus)$ generator have the same statistical characteristics, their results of this test should apply to any subset of bits in a random number sequence produced by this generator. This is in accordance with our results, where no correlations were found in the 31 bits of R250 (see Table 凹).

The initialization of R250 deserves a more detailed discussion. Already Kirkpatrick and Stoll [29] have pointed out that the algorithm $\operatorname{GFSR}(250,103, \oplus)$ requires a careful initialization. Our results in Table 5 clearly show this to be true as the results for R250 initialized with RAN3 show correlations in the most significant bits, an obvious consequence of the bad quality of RAN3. It is particularly important to notice, that these correlations once present seem to persist in R250.

Finally, for testing purposes we also realized our own Fortran implementation of LCG $\left(69069,1,2^{32}\right)$ in double precision accuracy. In this implementation, whenever the sign bit equalled one it was flipped to zero. Thus, the random numbers remained between zero and $2^{31}-1$. This implementation produced exactly the same sequence as RAND on a Convex C3840. Another possible implementation of the same algorithm was then realized in such a way that whenever the sign bit equalled one, the whole computer word was shifted to the right (with periodic boundary conditions) until a zero was obtained for the sign bit. When bit level tests were done for this implementation, all 31 bits failed. This dramatically highlights the effect of a poor implementation on the performance of the same algorithm.

\subsection{Visual tests}

The two dimensional distribution of 20000 random number pairs $\left(x_{i}, x_{i+1}\right)$ from GGL, RAND and R250 is shown in Figs. $1(a)-(f)$. When plotted on the scale from zero to one (Figs. $1(a),(c),(e)$ ), no generator shows any discernible structure. However, when the 20000 random numbers are plotted on an expanded scale (Figs. $1(b),(d),(f))$ one can clearly see the random numbers ordering on planes in the cases of GGL and RAND. This kind of behavior is expected for LCG generators [40], and the results are in accordance with the spectral test of Section 3.3. We note that no structure on other generators was observed on this scale. 
In Figs. 2(a) and (b), we depict subsequent random numbers in binary form on a $124 \times 124$ matrix from our best implementations of GGL and R250, respectively. Although the former showed clear lattice structure in the test above, in binary form it is very difficult to find any differences between these two generators. More quantitative tests of the bit maps shown here are in progress [59]. When we further compare the binary representations of R250 with different initializations, the visual tests corroborate the findings of the qualitative tests: In Fig. 2(b), R250 is initialized with GGL with double precision modulo operation, returning integers. However, if we initialize R250 with real numbers from GGL implemented in single precision, the result is catastrophic as seen in Fig. 3. Clearly this is an improper way to implement MLCG $\left(16807,2^{31}-1\right)$ in 32 bit word computers. It is interesting to note, that already Lewis et al. [36] pointed out the need to use double precision accuracy with the assembly implementation of MLCG $\left(16807,2^{31}-1\right)$.

The visualization of the difference between random numbers (test (iii)) and the gap test visualization (test (iv)) gave rather inconclusive results and thus yielded no further insight to the properties of the generators.

Finally, a problem was encountered with the decoding program which was included with the physical PURAN II random numbers [52]. When used to extract random numbers in floating point representation, we found that it produced numbers which fell on planes similarly to the linear congruential generators, although PURAN II passed all bit level tests. However, when using the decoding algorithm in integer format the problem disappeared.

\subsection{Speed of generators}

We tested the computational speed of the eight generators both on a Cray X-MP/432 and a Convex C3840. All generators were compiled in two ways: first, only scalar optimization was allowed and second, also vectorization was allowed. The testing was done for sequences of lengths $n=1,10,100,1000,10000$ and 100000 .

Results are in Table 6 for $n=1$ and $n=1000$ in units of microseconds $(\mu \mathrm{s})$ per random number call. The speedup for longer sequences $(n>1000)$ per random number call is nonexistent. First, Cray's own generator RANF was always the fastest on it which indicates a successful implementation in this sense. Other generators are almost equally fast for short sequences, except for R250. On the other hand, the performance for longer sequences is fastest for R250 and G05FAF if vectorizing 
is allowed. The code for RAN3 as given in Numerical Recipes [51] was incompatible with Cray and is thus omitted from its performance results. 


\title{
5 Summary and Discussion
}

\author{
"The whole history of \\ pseudorandom number generation \\ is riddled with myths and extrapolations \\ from inadequate examples. \\ A healthy dose of sceptisism is needed \\ in reading the literature." \\ B. D. RIPLEY
}

In this work, we have carried out an extensive test program of a collection of random number generators, which are commonly used in the applications of physics. These include public domain programs GGL, RANMAR, RAN3, RCARRY and R250, a library subroutine G05FAF, and manufacturer installed routines RAND and RANF. Also, a sequence of random numbers produced from physical noise has been included for purposes of comparison. Our test bench consists of standard statistical tests, bit level tests and qualitative visual tests. If we use the first two quantitative tests as criteria, three of the generators, namely GGL, G05FAF, and R250 display an overall best performance in all tests, and could thus be recommended for most applications. They fail statistical tests only once, and produce 31 "good" bits. Other generators show somewhat less convincing performance in one or more test category, although RANF performs very well in statistical tests. If the least significant bits are not important for the application, both RANF and RANMAR are good choices. On the other hand, the clear bit level correlations of RAN3 and poor statistical properties of RCARRY suggest problems in these generators [38]. Finally, RAND suffers from an overall lackluster performance. In Table 7 we show a qualitative summary of the performance of all of the generators in statistical and bit level tests.

Our results also demonstrate the existence of two fundamental problems which may plague some random number generators. First, a bad implementation of a generator algorithm may cause total corruption of the output, as we have demonstrated for GGL and RAND. Second problem concerns the initialization of generators such as R250, which require several seed values. This issue has received relatively little attention in the past, but our results in Section 4.3 demonstrate that as a result of a bad initialization, correlations in the seeds of R250 transform into the generated random number sequences. Thus even a good generator can be corrupted by careless use.

Despite the extensive test program presented here, there may still exist correlations 
which may be of significance. To this end, direct physical, application specific tests of various generators play an important role and have been conducted in some special cases [29, 28, 49, 46, 14, 23, 59]. These tests are of particular importance in Monte Carlo simulations, where physical systems may be very sensitive to spatial correlations. In particular, it has recently been suggested that biased results in Monte Carlo simulations of the Ising model [14] and self - avoiding random walks [23] result from yet undetected correlations present in the $\operatorname{GFSR}(250,103, \oplus)$ algorithm $(\mathrm{R} 250$ here). In both cases, special simulation algorithms were used. In Ref. [14] the authors suggest that bit level correlations in the most significant bits of this generator are responsible for this. However, our results of Sec. 4.3 do not lend support to this claim, since no discernible correlations exist up to at least 50 numbers apart. We have in fact recently extended the bit level tests to check correlations up to about 1000 numbers apart, but find no correlations for our R250 [59]. Results of Ref. [14] thus remain unexplained at the moment. On the other hand, Ref. 223 claims to confirm these anomalous correlations for $\operatorname{GFSR}(250,103, \oplus)$, and finds poor performance also for $\operatorname{LF}(55,24,+)$ (our RAN3 is $\operatorname{LF}(55,24,-)$ ). RAN3 spectacularly fails our bit level tests, which probably explains results of Ref. [23 for the lagged Fibonacci generator. However, concerning $\operatorname{GFSR}(250,103, \oplus)$ Ref. [23] goes as far as to reinforce the claim 42] that "shift register generators using XOR's are among the worst random number generators and should never have been used". Based on our test results this is a somewhat unfair statement, since R250 when properly implemented and initialized certainly performs well enough for many applications. However, we agree with Ref. [14] on the need of careful physical tests before a "good quality" generator is chosen for a given application. To unravel possible anomalous correlations in R250, a new generation of test methods is clearly needed since no test carried out here can support this claim. Work in this direction is currently underway [59].

\section{Acknowledgments}

We would like to thank Juha Haataja, Jukka Helin, Pentti Huuhtanen, Kimmo Kaski, Juhani Käpyaho, Petri Laurikainen, Jussi Rahola, Robert Swendsen, and Jukka Vanhala for useful discussions and Fred James and Manfred Richter for correspondence. Veikko Nyfors of Cray Finland Oy, Greg Astfalk of Convex Computer Corporation and Julie Gulla of Mathworks Inc. have provided information of the random number generators used in their products. Aarno Hauru, Pekka Kytölaakso, 
Klaus Lindberg and Juha Ruokolainen have given valuable technical assistance. The Finnish Center for Scientific Computing and Tampere University of Technology have generously provided the computing resources. This research was partially supported by the Academy of Finland and the Foundation of Tampere University of Technology. 
E-mail addresses: Ilpo.Vattulainen@csc.fi, Kari.Kankaala@csc.fi, jukkas@ee.tut.fi, and ala@phcu.helsinki.fi

\section{References}

[1] E. Aarts And J. Korst, Simulated Annealing and Boltzmann Machines, A Stochastic Approach to Combinatorial Optimization and Neural Computing (John Wiley \& Sons, Chichester, 1989).

[2] N. S. Altman, SIAM J. Sci. Stat. Comput. 9, 941 (1988).

[3] S. L. Anderson, SIAM Review 32, 221 (1990).

[4] L. M. Berliner, Stat. Science 7, 69 (1992).

[5] K. Binder, in Monte Carlo Methods in Condensed Matter Physics, edited by K. Binder (Springer - Verlag, Berlin, 1992).

[6] H. S. Bright And R. L. Enison, Computing Surveys 11, 357 (1979).

[7] B. J. Collings and G. B. Hembree, J. ACM 33, 706 (1986).

[8] A. Compagner and A. Hoogland, J. Comp. Phys. 71, 391 (1987).

[9] A. Compagner, Am. J. Phys. 59, 700 (1991).

[10] A. Compagner, J. Stat. Phys. 63, 883 (1991).

[11] R. R. Coveyou and R. D. MacPherson, J. ACM 14, 100 (1967).

[12] D. W. Davies And W. L. PRICE, Security for Computer Networks, An Introduction to Data Security in Teleprocessing and Electronic Funds Transfer, 2nd ed. (John Wiley \& Sons, Chichester, 1989).

[13] E. J. Dudewicz And T. G. Ralley, in The Handbook of Random Number Generation and Testing with TESTRAND Computer Code, Am. Series in Math. and Manag. Sci., Vol. 1 (American Science Press, Ohio, USA, 1981).

[14] A. M. Ferrenberg, D. P. Landau and Y. J. Wong, Phys. Rev. Lett. 69, 3382 (1992).

[15] G. S. Fishman and L. R. Moore III, J. Am. Stat. Assoc. 77, 129 (1982). 
[16] G. S. Fishman and L. R. Moore III, SIAM J. Sci. and Stat. Comp. 7, 24 (1986).

[17] G. S. Fishman, Math. Comp. 54, 331 (1990).

[18] M. Fushimi and S. Tezuka, Comm. ACM 26, 516 (1983).

[19] M. Fushimi, Inform. Proc. Lett. 16, 189 (1983).

[20] M. Fushimi, SIAM J. Comp. 17, 89 (1988).

[21] S. Garpman and J. Randrup, Comp. Phys. Comm. 15, 5 (1978).

[22] J. E. Gentle, J. Comp. Appl. Math. 31, 119 (1990).

[23] P. Grassberger, Wuppertal University preprint WUB 93-03 (1993).

[24] J. Helin, unpublished work (1985).

[25] J. R. Heringa, H. W. J. Blöte and A. Compagner, Int. J. Mod. Phys. C 3, 561 (1992).

[26] N. Ito, M. Kikuchi, Y. Okabe, Cologne University preprint (1992), heplat@ftp.scri.fsu.edu 9302002.

[27] F. James, Comp. Phys. Comm. 60, 329 (1990).

[28] C. Kalle and S. Wansleben, Comp. Phys. Comm. 33, 343 (1984).

[29] S. Kirkpatrick and E. P. Stoll, J. Comp. Phys. 40, 517 (1981).

[30] D. E. Knuth, The Art of Computer Programming, Volume 2: Seminumerical Algorithms, 2nd ed. (Addison-Wesley, Reading, Massachusetts, 1981).

[31] M. van Lambalgen, J. Symbolic Logic 52, 725 (1987).

[32] G. P. Learmonth and P. A. W. Lewis, in Computer Science and Statistics: 7 th Annual Symposium on the interaface, edited by W. J. Kennedy, (Statistical Laboratory, Iowa State University, Ames, Iowa, 1973) p. 163.

[33] P. L'ECuyer, Comm. ACM 31, 742 (1988).

[34] P. L'ECuyer, Comm. ACM 33, 86 (1990).

[35] D. H. Lehmer, in Proc. 2nd Symp. on Large-Scale Digital Calculating Machinery (Harvard University Press, Cambridge, 1951), p. 141. 
[36] P. A. Lewis, A. S. Goodman and J. M. Miller, IBM Syst. J. 8, 136 (1969).

[37] T. G. Lewis and W. H. Payne, J. Assoc. Comput. Mach. 20, 456 (1973).

[38] Recently, M. Lücher (unpublished) has noted that there's a bug in the implementation of RCARRY, which affects its output. We have repeated the gap tests for the corrected version, but find no significant improvement in the statistical properties.

[39] M. D. Maclaren and G. Marsaglia, J. Assoc. Comput. Mach. 12, 83 (1965).

[40] G. Marsaglia, Proc. of the Nat. Acad. Sci. 61, 25 (1968).

[41] G. Marsaglia, in Applications of Number Theory to Numerical Analysis, edited by S. K. Zaremba (Academic Press, New York, 1972), p. 249.

[42] G. A. Marsaglia, in Computer Science and Statistics: The Interface, edited by L. Billard, (Elsevier, Amsterdam, 1985) p. 3.

[43] G. Marsaglia and A. Zaman, Stat. \& Prob. Lett. 8, 329 (1990).

[44] G. Marsaglia, B. Narasimhan and A. Zaman, Comp. Phys. Comm. 60, 345 (1990).

[45] A. De Matteis and S. Pagnutti, Parallel Computing 13, 193 (1990).

[46] A. Milchev, K. Binder and D. W. Heermann, Z. Phys. B 63, 521 (1986).

[47] H. Niederreiter, SiAM J. Sci. Stat. Comput. 8, 1035 (1987).

[48] S. K. Park and K. W. Miller, Comm. ACM 31, 1192 (1988).

[49] J. Paulsen, J. Stat. Comput. Simul. 19, 23 (1984).

[50] W. H. Payne and K. L. McMillen, Comm. ACM 21, 259 (1978).

[51] W. H. Press, B. P. Flannery, S. A. Tenkolsky and W. T. VetterLING, Fortran version (Cambridge University Press, 1989) p. 198.

[52] M. RICHTER, private communication. The random numbers by PURAN II are available from an anonymous ftp site at dfv.rwth-aachen.de.

[53] L. Schrage, ACM Trans. Math. Soft. 5, 132 (1979). 
[54] R. C. Tausworthe, Math. Comp. 19, 201 (1965).

[55] S. Tezuka, Comm. ACM 30, 731 (1987).

[56] S. TezukA, J. Assoc. Comp. Mach. 34, 939 (1987).

[57] J. P. R. Tootill, W. D. Robinson and A. G. Adams, J. Assoc. Comput. Mach. 18, 381 (1971).

[58] I. Vattulainen, K. Kankaala, J. Saarinen and T. Ala-Nissila, CSC Research Report R05/92 (Centre for Scientific Computing, Espoo, Finland 1992). In Finnish.

[59] I. Vattulainen, K. Kankaala, J. Saarinen, and T. Ala-Nissila, to be published.

[60] N. Zierler and J. Brillhart, Inform. and Control 13, 541 (1968).

[61] K. Zheng, C.-H. Yong, D.-Y. Wei, IEEE Computer 24, 8 (1991).

[62] N. Zierler, Inform. and Control 15, 67 (1969).

[63] Convex Fortran Guide, 1st edition (Convex Computer Corp., Richardson, USA, 1991), p. 553.

[64] Cray Unicos, Math and Scientific Library Reference Manual, SR-2081 6.0 (Cray Research Inc., USA, 1991).

[65] IBM Subroutine Library - Mathematics (User's Guide program number 5736XM7, 1971).

[66] IMSL Stat/Library User's Manual 3 (IMSL, Houston, Texas, 1989), p. 945.

[67] MATLAB User's Guide, PRO-MATLAB for VAX/VMS Computers (The MathWorks Inc., South Natick, MA, 1991), p. 3-158; J. Gulla, private communication.

[68] NAG Fortran Library Manual, Mark 14, 7 (Numerical Algorithms Group Inc., 1990). 


\section{Table captions}

\section{Table 1}

TABLE 1. Parameters used in the standard tests. $n$ is the length of the random number sequence and $N$ is the number of times the test was repeated for the Kolmogorov - Smirnov test. Other parameters are described in the text.

\section{Table 2}

TABle 2. Results of the statistical tests. Depicted numbers are the values for the descriptive levels $\delta^{+}$and $\delta^{-}$from the Kolmogorov - Smirnov test variables $K^{+}$and $K^{-}$, and $R_{\delta}$ and $R_{K}$ denote average goodness values, as defined in the text (with tests 1 and 2 excluded). The data for $R$ 's comes from the first run only. A generator was considered to fail the test if the descriptive level was less than 0.05 or more than 0.95. Single, double and triple consequtive failures are indicated by single, double, and bold lines, respectively.

\section{Table 3}

TABLE 3. Results of the spectral test for linear congruential generators. See text for details.

\section{Table 4}

TABlE 4. Results of the bit level $d$-tuple and rank tests. The bits marked failed have failed the test twice. See text for details.

\section{Table 5}

TABLE 5. Results of $d$-tuple and rank tests for R250 initialized with RAN3.

\section{Table 6}

TABLE 6. Absolute speeds of the generators on a Cray X-MP/432 EA and a Convex C3840. S denotes compiling when only scalar optimization was allowed and V when also vectorizing was allowed. The timings are in units of microseconds per random number call. RANF could only be tested on Cray and RAND on Convex. RAN3 produced erroneous results on Cray.

\section{Table 7}

TABLE 7. A summary of the performance of the tested generators in statistical and bit level tests. For statistical tests, plus denotes at least one case of one consequtive 
failure, zero at least one case of two consequtive failures, and minus at least one case of three consequtive failures. For bit level tests, plus denotes an impeccable performance, zero the failure of some of the least significant bits, and minus the failure of more significant bits for RAND and RAN3. See text for more details. 


\section{Figure Captions}

\section{Figure 1}

FIG. 1. Spatial distribution of 20000 random number pairs in two dimensions on a unit square as generated by GGL $(a),(b)$, RAND $(c),(d)$ and R250 $(e),(f)$. The second figure in each case has a greatly expanded scale on the $x$ axis.

\section{Figure 2}

FIG. 2. 31 bit binary representations of random numbers produced by GGL $(a)$ and R250 (b) on a $124 \times 124$ matrix.

\section{Figure 3}

FIG 3. Binary representations of random numbers produced by R250 when initialized with GGL in single precision mode. 


\begin{tabular}{|l|l|r|r|lll|}
\hline \multicolumn{2}{|c|}{ Test } & \multicolumn{1}{|c|}{$n$} & \multicolumn{1}{c|}{$N$} & \multicolumn{3}{|c|}{ Other parameters } \\
\hline \hline$(1)$ & $\chi^{2}$ & 100000 & 10000 & $\nu=256$ & & \\
$(2)$ & $\chi^{2}$ & 10000 & 10000 & $\nu=128$ & & \\
$(3)$ & Serial test & 100000 & 1000 & $d=2$ & $\nu=100$ & \\
$(4)$ & Serial test & 100000 & 1000 & $d=3$ & $\nu=20$ & \\
$(5)$ & Serial test & 100000 & 1000 & $d=4$ & $\nu=10$ & \\
$(6)$ & Gap test & 25000 & 1000 & $\alpha=0$ & $\beta=0.05$ & $l=30$ \\
$(7)$ & Gap test & 25000 & 1000 & $\alpha=0.45$ & $\beta=0.55$ & $l=30$ \\
$(8)$ & Gap test & 25000 & 1000 & $\alpha=0.95$ & $\beta=1$ & $l=30$ \\
$(9)$ & Maximum of $t$ & 2000 & 1000 & $t=5$ & & \\
$(10)$ & Maximum of $t$ & 2000 & 1000 & $t=3$ & & \\
$(11)$ & Collision test & 16384 & 1000 & $d=2$ & $s=1024$ & \\
$(12)$ & Collision test & 16384 & 1000 & $d=4$ & $s=32$ & \\
$(13)$ & Collision test & 16384 & 1000 & $d=10$ & $s=4$ \\
$(14)$ & Run test & 100000 & 1000 & $l=6$ & & \\
\hline
\end{tabular}

Table 1: 
Table 2: 


\begin{tabular}{|c|c|c|c|c|c|c|c|c|c|c|}
\hline \multirow{2}{*}{$d$} & \multicolumn{2}{|c|}{ RAND } & \multicolumn{2}{c|}{ GGL } & \multicolumn{2}{c|}{ G05FAF } & \multicolumn{2}{c|}{ RANF 1 } & \multicolumn{2}{c|}{ RANF 2 } \\
\cline { 2 - 10 } & $\kappa_{d}$ & $\lambda_{d}$ & $\kappa_{d}$ & $\lambda_{d}$ & $\kappa_{d}$ & $\lambda_{d}$ & $\kappa_{d}$ & $\lambda_{d}$ & $\kappa_{d}$ & $\lambda_{d}$ \\
\hline \hline 2 & 0.9250 & 15.991 & 0.3375 & 14.037 & 0.8423 & 29.356 & 0.8269 & 22.8295 & 0.6499 & 22.482 \\
3 & 0.7890 & 10.492 & 0.4412 & 9.319 & 0.7640 & 19.533 & 0.7416 & 15.069 & 0.7705 & 15.124 \\
4 & 0.7548 & 7.844 & 0.5752 & 7.202 & 0.8472 & 14.260 & 0.3983 & 10.422 & 0.7071 & 11.250 \\
5 & 0.8041 & 6.386 & 0.7361 & 6.058 & 0.7838 & 11.348 & 0.7307 & 9.047 & 0.3983 & 8.172 \\
6 & 0.2990 & 3.959 & 0.6454 & 4.903 & 0.6333 & 9.209 & 0.6177 & 7.339 & 0.6282 & 7.364 \\
7 & 0.4075 & 3.705 & 0.5711 & 4.049 & 0.5540 & 8.382 & 0.6670 & 6.416 & 0.2375 & 4.926 \\
8 & 0.5762 & 3.705 & 0.6096 & 3.661 & 0.6597 & 7.271 & 0.5642 & 5.424 & 0.2135 & 4.022 \\
\hline
\end{tabular}

Table 3: 


\begin{tabular}{|c|c|c|c|}
\hline $\begin{array}{l}\text { Random } \\
\text { number } \\
\text { generator }\end{array}$ & $\begin{array}{l}\text { Failing bits } \\
\text { in the } \\
d \text {-tuple test }\end{array}$ & $\begin{array}{l}\text { Failing bits } \\
\text { in the rank } \\
\text { test }\end{array}$ & $\begin{array}{l}\text { Comments of } \\
\text { implementation } \\
\text { and initialization }\end{array}$ \\
\hline GGL & none & none & $\begin{array}{l}\text { double precision mode } \\
\text { (return integers) }\end{array}$ \\
\hline RAND & $13-31$ & $18-31$ & real mode \\
\hline RANF & $29-45$ & $24,31-45$ & real mode \\
\hline G05FAF & none & none & double precision mode \\
\hline $\mathrm{R} 250$ & none & none & $\begin{array}{l}\text { integer mode, initialized } \\
\text { with GGL in double precision }\end{array}$ \\
\hline RAN3 & $1-5,25-30$ & $1-5,26-30$ & integer mode \\
\hline RANMAR & $25-31$ & $25-31$ & real mode \\
\hline RCARRY & $25-31$ & $25-31$ & real mode \\
\hline PURAN II & none & none & integer mode \\
\hline
\end{tabular}

Table 4: 


\begin{tabular}{|l|l|l|}
\hline $\begin{array}{l}\text { Random } \\
\text { number } \\
\text { generator }\end{array}$ & $\begin{array}{l}\text { Failing bits } \\
\text { in the } \\
d \text {-tuple test }\end{array}$ & $\begin{array}{l}\text { Failing bits } \\
\text { in the } \\
\text { rank test }\end{array}$ \\
\hline \hline R250 & $1-2,27-31$ & $1,27-31$ \\
RAN3 & $1-5,25-30$ & $1-5,26-30$ \\
\hline
\end{tabular}

Table 5: 


\begin{tabular}{|l|c|c|c|c|c|}
\hline \multirow{2}{*}{ Generator } & \multirow{2}{*}{ Optimization } & \multicolumn{2}{|c|}{ Cray } & \multicolumn{2}{c|}{ Convex } \\
\cline { 3 - 6 } & & $n=1$ & $n=1000$ & $n=1$ & $n=1000$ \\
\hline \hline GGL & $\mathrm{S}$ & 2.218 & 2.731 & 4.420 & 2.379 \\
& $\mathrm{~V}$ & 2.465 & 2.029 & 5.676 & 2.381 \\
\hline RAND & $\mathrm{S}$ & - & - & 4.446 & 4.582 \\
& $\mathrm{~V}$ & - & - & 6.661 & 4.369 \\
\hline RANF & $\mathrm{S}$ & 1.466 & 1.582 & - & - \\
& $\mathrm{V}$ & 1.536 & 0.020 & - & - \\
\hline G05FAF & $\mathrm{S}$ & 4.556 & 0.422 & 4.384 & 0.571 \\
& $\mathrm{~V}$ & 4.442 & 0.365 & 6.321 & 0.559 \\
\hline R250 & $\mathrm{S}$ & 260.0 & 1.672 & 126.7 & 1.094 \\
& $\mathrm{~V}$ & 10.88 & 0.055 & 55.87 & 0.476 \\
\hline RAN3 & $\mathrm{S}$ & 4.711 & - & 3.987 & 2.177 \\
& $\mathrm{~V}$ & 3.563 & - & 4.881 & 1.608 \\
\hline RANMAR & $\mathrm{S}$ & 7.132 & 3.407 & 5.672 & 1.932 \\
& $\mathrm{~V}$ & 4.801 & 1.053 & 5.742 & 1.508 \\
\hline RCARRY & $\mathrm{S}$ & 6.486 & 2.455 & 4.956 & 1.211 \\
& $\mathrm{~V}$ & 3.962 & 0.728 & 4.537 & 0.899 \\
\hline
\end{tabular}

Table 6: 


\begin{tabular}{|l|c|c|c|c|c|c|c|c|}
\hline \multirow{2}{*}{$\begin{array}{l}\text { Test } \\
\text { method }\end{array}$} & \multicolumn{7}{|c|}{ Random number generator } \\
\cline { 2 - 9 } & GGL & RAND & RANF & G05FAF & R250 & RAN3 & RANMAR & RCARRY \\
\hline \hline Standard tests & + & 0 & + & + & + & 0 & - & - \\
Bit level tests & + & - & 0 & + & + & - & 0 & 0 \\
\hline
\end{tabular}

Table 7: 


\begin{tabular}{|c|c|c|c|c|c|c|c|c|c|c|c|c|c|c|c|c|}
\hline \multirow[b]{2}{*}{ Test } & \multicolumn{2}{|c|}{ GGL } & \multicolumn{2}{|c|}{ RAND } & \multicolumn{2}{|c|}{ RANF } & \multicolumn{2}{|c|}{ G05FAF } & \multicolumn{2}{|c|}{ R250 } & \multicolumn{2}{|c|}{ RAN3 } & \multicolumn{2}{|c|}{ RANMAR } & \multicolumn{2}{|c|}{ RCARRY } \\
\hline & $K^{+}$ & $K^{-}$ & $K^{+}$ & $K^{-}$ & $K^{+}$ & $K^{-}$ & $K^{+}$ & $K^{-}$ & $K^{+}$ & $K^{-}$ & $K^{+}$ & $K^{-}$ & $K^{+}$ & $K^{-}$ & $K^{+}$ & $K^{-}$ \\
\hline 1 & 0.205 & 0.249 & 0.829 & 0.490 & 0.562 & 0.655 & 0.609 & 0.049 & 0.112 & 0.518 & 0.709 & 0.748 & 0.963 & 0.202 & 0.194 & 0.639 \\
\hline 2 & 0.718 & 0.907 & 0.043 & 0.982 & 0.592 & 0.421 & 0.391 & 0.778 & 0.907 & 0.713 & 0.966 & 0.149 & 0.219 & 0.490 & 0.690 & 0.213 \\
\hline 3 & 0.672 & 0.380 & 0.228 & 0.895 & 0.924 & 0.039 & 0.700 & 0.570 & 0.678 & 0.292 & 0.798 & 0.903 & 0.419 & 0.482 & 0.070 & 0.570 \\
\hline 4 & 0.642 & 0.238 & 0.280 & 0.541 & 0.242 & 0.667 & 0.702 & 0.095 & 0.256 & 0.573 & 0.553 & 0.762 & 0.262 & 0.666 & 0.175 & 0.494 \\
\hline 5 & 0.780 & 0.465 & 0.134 & 0.697 & 0.582 & 0.788 & 0.494 & 0.380 & 0.991 & 0.103 & 0.734 & 0.040 & 0.031 & 0.929 & 0.961 & 0.274 \\
\hline 6 & 0.900 & 0.649 & 0.559 & 0.479 & 0.562 & 0.472 & 0.609 & 0.143 & 0.544 & 0.079 & 0.049 & 0.927 & 0.545 & 0.486 & 0.000 & 1.000 \\
\hline 7 & 0.976 & 0.100 & 0.987 & 0.344 & 0.368 & 0.582 & 0.957 & 0.018 & 0.343 & 0.929 & 0.379 & 0.484 & 0.795 & 0.274 & 0.169 & 0.909 \\
\hline 8 & 0.768 & 0.048 & 0.380 & 0.816 & 0.630 & 0.305 & 0.986 & 0.161 & 0.332 & 0.415 & 0.494 & 0.664 & 0.492 & 0.317 & 0.000 & 1.000 \\
\hline $9^{+}$ & 0.490 & 0.331 & 0.326 & 0.981 & 0.900 & 0.115 & 0.255 & 0.133 & 0.294 & 0.365 & 0.761 & 0.029 & 0.661 & 0.311 & 0.436 & 0.968 \\
\hline $9^{-}$ & 0.887 & 0.000 & 0.938 & 0.018 & 0.947 & 0.108 & 0.967 & 0.108 & 0.951 & 0.433 & 0.926 & 0.001 & 0.735 & 0.164 & 0.989 & 0.006 \\
\hline $10^{+}$ & 0.985 & 0.052 & 0.107 & 0.795 & 0.214 & 0.788 & 0.832 & 0.508 & 0.718 & 0.356 & 0.966 & 0.704 & 0.948 & 0.048 & 0.145 & 0.540 \\
\hline $10^{-}$ & 0.864 & 0.028 & 0.981 & 0.001 & 1.000 & 0.000 & 0.998 & 0.000 & 0.750 & 0.162 & 0.999 & 0.101 & 0.684 & 0.000 & 0.999 & 0.026 \\
\hline 11 & 0.161 & 0.561 & 0.974 & 0.076 & 0.906 & 0.146 & 0.105 & 0.653 & 0.807 & 0.670 & 0.049 & 0.769 & 0.977 & 0.093 & 0.069 & 0.553 \\
\hline 12 & 0.654 & 0.159 & 0.888 & 0.071 & 0.093 & 0.426 & 0.175 & 0.909 & 0.720 & 0.237 & 0.847 & 0.119 & 0.799 & 0.350 & 0.854 & 0.102 \\
\hline 13 & 0.411 & 0.551 & 0.102 & 0.137 & 0.363 & 0.607 & 0.430 & 0.413 & 0.480 & 0.347 & 0.809 & 0.069 & 0.654 & 0.344 & 0.383 & 0.417 \\
\hline 14 & 0.073 & 0.755 & 0.356 & 0.946 & 0.276 & 0.297 & 0.124 & 0.637 & 0.368 & 0.226 & 0.785 & 0.154 & 0.268 & 0.375 & 0.339 & 0.851 \\
\hline$R$ & \multicolumn{2}{|c|}{$2.52 \%$} & \multicolumn{2}{|c|}{$6.62 \%$} & \multicolumn{2}{|c|}{$4.86 \%$} & \multicolumn{2}{|c|}{$0.62 \%$} & \multicolumn{2}{|c|}{$2.47 \%$} & \multicolumn{2}{|c|}{$7.74 \%$} & \multicolumn{2}{|c|}{$6.30 \%$} & \multicolumn{2}{|c|}{$7.66 \%$} \\
\hline
\end{tabular}

